\title{
Thermodynamic Properties of Organic Compounds. 2. Combustion and Sublimation Enthalpies of 2,4,6-Trimethylbenzonitrile $\mathbf{N}$-Oxide
}

\author{
William E. Acree, Jr." \\ Department of Chemistry, University of North Texas, Denton, Texas 76203-5068
}

Vladimir V. Simirsky, Alexander A. Kozyro, Alexander P. Krasulln, and Gennady J. Kabo

Department of Chemistry, Byelorussian State University, Minsk, 220080 USSR

Michael L. Frenkel

Thermodynamics Research Center, Texas A\&M University, College Station, Texas 77843-3111

\begin{abstract}
Experlmental vapor pressures and enthalpy of combustion are reported for 2,4,6-trimethylbenzonitrlle $N$-oxide, along with the enthalpy of sublimation derived from the temperature dependence of the vapor pressure. Results of these measurements are used to compute standard enthalples of formation in the crystalline and gaseous states, $\Delta_{1} H_{m}{ }^{\circ}(\mathrm{cr})=49.25 \pm 3.54 \mathrm{~kJ} \cdot \mathrm{mol}^{-1}$ and $\Delta_{\mathrm{r}} H_{\mathrm{m}}{ }^{\circ}(\mathrm{g})$ $=137.0 \pm 4.0 \mathrm{~kJ} \cdot \mathrm{mol}^{-1}$, respectively.
\end{abstract}

\section{Introduction}

Nitrile $\mathrm{N}$-oxides are highly reactive organic compounds prepared from oxidation of aldoximes and dehydration of nitro compounds. Reported half-lives of aliphatic nitrile $\mathrm{N}$-oxides range from a few seconds to minutes before rearrangement to isocyanates or polymerization occurs. Aromatic nitrile $\mathrm{N}$-oxides are considerably more stable, particularly 1,4-dicyanobenzene di-N-oxide $(1,2)$, which can be stored for several months without noticeable degradation. Bulky substituents in the ortho positions of the benzene ring or on the $\alpha$-carbon aliphatic derivatives enhance stability. Sterically hindered nitrile $N$-oxides such as 2,6-dimethylbenzonitrile $N$-oxide and di-tert-butylacetonitrile $\mathrm{N}$-oxide are "permanently stable" at room temperature (3-5).

Chemical reactivity of the nitrile $\mathrm{N}$-oxides undoubtedly arises because of the ionic nature of the $\mathrm{N}-\mathrm{O}$ dative bond. Nuclear magnetic resonance ${ }^{13} \mathrm{C}$ chemical shift data for 2,4,6-trimethylbenzonitrile $\mathrm{N}$-oxide (6) suggest that the carbon atom carries substantial negative charge (i.e., the $\mathrm{R}-\mathrm{C}^{-}=\mathrm{N}^{+}=\mathrm{O}$ resonance form) and are in accord with published $X$-ray crystallographic bond distances for 4-methoxy-2,6-dimethylbenzonitrile $N$-oxide (7). Surprisingly, very few precise thermochemical quantities are available in the chemical literature for nitrile $\mathrm{N}$-oxides. Acree, Pilcher, and co-workers (8) previously reported the standard enthalpies of combustion and sublimation for 2,4,6-trimethylbenzonitrile $N$-oxide and 2,4,6-trimethoxybenzonitrile $N$-oxide as determined by static bomb calorimetric and "vacuum sublimation" drop microcalorimetric methods, respectively. Because of the scarcity of experimental data for this relatively novel class of compounds, we have undertaken a comprehensive thermodynamic study of 2,4,6-trimethylbenzonitrile $\mathrm{N}$-oxide, which includes both heat capacity and vapor pressure measurements, as well as redetermination of the enthalpy of combustion. In this short communication we report results from the combustion and vapor pressure measurements. The heat capacity data will be presented at a later date.

\footnotetext{
- To whom correspondence should be addressed.
}

\section{Experimental Methods}

2,4,6-Trimethylbenzonitrile $N$-oxide was prepared by the procedures outlined by Grundmann and Dean (5) and Beltrame et al. (9). Hydroxylamine hydrochloride was added to a hot solution of 2,4,6-trimethylbenzaldehyde in methanol, and the resulting aldoxime was converted to the nitrile $\mathbf{N}$-oxide with sodium hypobromite. The crude sample was further purified by three recrystallizations from a benzene-hexane mixture. Elemental analysis on the purified sample was in excellent agreement with calculated values. Anal. Found for $\mathrm{C}_{10} \mathrm{H}_{11} \mathrm{NO}$ (mass \%): C, 74.58; H, 6.94; N, 8.62. Calcd (mass \%): C, $74.51 ; H, 6.88 ; \mathrm{N}, 8.69$. The sample was stored in a cool, dark place for several weeks and later shipped to the Soviet Union via airmail during a period of heightened airport security.

Experimental procedures and instrumentation are described in detall elsewhere $(10-13)$. Brlefly, combustion energies were measured in a calorimeter with an isothermal jacket whose temperature was controlled to within $0.0015 \mathrm{~K}$. The calorimeter vessel temperature was measured with $100-\Omega$ platinum resistance thermometers and recorded on a digital voltmeter. The calorimeter has been automated in order to facilitate transfer of information from the digltal voltmeter to a microcomputer. An automation scheme enables one to vary the temperature intervals between measurements, warms the operator of the approaching ignition time, checks the success of ignition, and signals the end of measurements. The volume of the empty bomb was $0.3205 \mathrm{dm}^{3}$. The sample was ignited using a 10000 $\mu \mathrm{F}$ capacity bank discharged through a $0.05-\mathrm{mm}$ platinum wire $(14,15)$. All experiments were performed at an initial bomb pressure of $3.0 \mathrm{MPa}$. Oxygen was prepurifled from an organic admixture, carbon dioxide and water. The energy equivalent of the calorimeter $14889.9 \pm 6.4 \mathrm{~J} \cdot \mathrm{K}^{-1}$ was determined for the standard benzolc acld K-I with a specific energy of combustion of $26460.6 \pm 4.6 \mathrm{~J} \cdot \mathrm{g}^{-1}$.

To ascertain the completeness of combustion, a gravimetric technique was used to determine the amount of $\mathrm{CO}_{2}(\mathrm{~g})$, undissolved $\mathrm{NO}_{2}$, and in some tests $\mathrm{CO}$, with the sensitivity of indicator tubes being $1 \times 10^{-4}$ (volume percent). In the $\mathrm{CO}_{2}$ tests, an $\mathrm{MnO}_{2}$ tube was inserted into the absorption system to absorb nitrogen oxides that might interfere with the $\mathrm{CO}_{2}$ measurements (16). To reduce the effect of nitrogen oxides, the gravimetric analysis was performed after the contents were stored in the bomb for $24 \mathrm{~h}$ (17). As the accuracy of welghing the trap system for gravimetric analysis was lower than that of the sample mass measurement, $\Delta_{c} U_{m}{ }^{\circ}$ was calculated from the mass of the calorimetric sample. In a series of special experiments we established that nitric oxides formed in the combustion of 2,4,6-trimethylbenzonitrile $\mathrm{N}$-oxide during the main period had time to turn in the bomb into $\mathrm{HNO}_{3}$ solution; 
Table I. Experimental Evaluation of the Enthalpy of Combustion of 2,4,6-Trimethylbenzonitrile $\boldsymbol{N}$-Oxide ${ }^{a}$

\begin{tabular}{cccccccc}
\hline run no. & $m_{0} / \mathrm{g}$ & $\alpha$ & $\Delta T / \mathrm{K}$ & $q_{\mathrm{ign}} / \mathrm{J}$ & $q_{\mathrm{HNO}_{3}{ }^{b} / \mathrm{J}}$ & $\sum q / \mathrm{J}$ & $-\Delta_{\mathrm{c}} U_{\mathrm{m}}{ }^{\circ} / m_{0} /\left(\mathrm{J} \cdot \mathrm{g}^{-1}\right)$ \\
\hline 1 & 0.52819 & 0.00100 & 1.22410 & 2.0 & 31.0 & 9.2 & 34428.1 \\
3 & 0.48929 & 0.00104 & 1.13443 & 2.0 & 28.6 & 8.4 & 34442.8 \\
4 & 0.48503 & 0.00108 & 1.12486 & 2.0 & 28.6 & 8.3 & 34451.6 \\
& 0.46127 & 0.00107 & 1.06959 & 2.0 & 28.0 & 7.8 & 34444.6 \\
& & & & & & & $34441.8 \pm 15.6(\mathrm{av})$
\end{tabular}

${ }^{a}$ Density of the solid at $293.15 \mathrm{~K}$ is $1.1565 \mathrm{~g} \cdot \mathrm{cm}^{-3} .{ }^{b}$ Estimated uncertainty is $\pm 0.1 \mathrm{~J}$.

that is why the correction for $\mathrm{HNO}_{3}$ generation was determined by the titration results extrapolated to the end point of the main period. The extrapolated value of the volume of $0.01 \mathrm{~mol} \cdot \mathrm{dm}^{-3}$ of alkali hydroxide used for titration was below $0.5 \mathrm{~cm}^{3}$. The Washburn corrections were estimated using the technique suggested by Prosen (18) with regard to recommendations given in ref 19.

Vapor pressures were measured between ca. 300 and 340 $K$ by an integral Knudsen effusion method $(12,13)$. The diameter of the effusion hole in the nickel membrane was $0.82543 \pm 0.0035 \mathrm{~mm}$ and the Knudsen constant, KS, was $50.4406 \times 10^{-8} \mathrm{~m}^{2}$. Calculated vapor pressures are based upon the assumption that the true molecular mass of the gaseous species is that of the monomer, $161.203 \mathrm{~g} \cdot \mathrm{mol}^{-1}$, which should be valid under these experimental conditions. Any polymerization product that could conceivably be formed from thermal/photochemical degradation of 2,4,6-trimethylbenzonitrile $\mathrm{N}$-oxide is expected to have a much higher molecular mass and thus would be extremely nonvolatile.

\section{Results and Discussion}

Combustion results are listed in Table I; $m_{0}$ is the mass of 2,4,6-trimethylbenzonitrile $N$-oxide determined in vacuum, $\Delta T$ is the corrected temeprature rise; $\alpha$ is the cooling constant of the calorimeter; $\sum q$ denotes the sum of the Washburn corrections; $q_{\operatorname{lgn}}$ is the ignition contribution to the observed; and $g_{\mathrm{HNO}_{3}}$ refers to the correction for production of nitric acid, which was subtracted from the observed heat. Errors in $\Delta_{c} U_{m}^{\circ}$ were computed as a product of standard deviation and Student coefficient for four independent determinations at the $95 \%$ confidence limit. Measurements on a smaller sample purified by vacuum sublimation gave essentially an identical $\Delta_{\mathrm{c}} U_{\mathrm{m}}{ }^{\circ}$ value but is not included in the statistical treatment. Standard molar enthalpies of combustion and formation in the crystalline state are $\Delta_{\mathrm{c}} H_{\mathrm{m}}{ }^{\circ}(\mathrm{cr})=-(5556.45 \pm 2.51) \mathrm{kJ} \cdot \mathrm{mol}^{-1}$ and $\Delta_{\mathrm{f}} H_{\mathrm{m}}{ }^{\circ}(\mathrm{cr})=49.25 \pm 3.54 \mathrm{~kJ} \cdot \mathrm{mol}^{-1}$, respectively. To derive this latter quantity, the standard molar enthalpies of formation for $\mathrm{H}_{2} \mathrm{O}(\mathrm{l}),-(285.83 \pm 0.04) \mathrm{kJ} \cdot \mathrm{mol}^{-1}$, and for $\mathrm{CO}_{2}(\mathrm{~g}),-(393.51$ $\pm 0.13) \mathrm{kJ} \cdot \mathrm{mol}^{-1}$, were used. The calculated enthalpy of formation differs only slightly from the published value of $55.9 \pm$ $1.5 \mathrm{~kJ} \cdot \mathrm{mol}^{-1}$ determined by Acree et al. (8).

Vapor pressures for 2,4,6-trimethylbenzonitrile $N$-oxide at temperatures between 300 and $345 \mathrm{~K}$ are tabulated in Table II for the sample as received via airmail (sample I) and after subsequent purification by vacuum sublimation (sample II). Experimental values can be described by the following mathematical expressions

sample I

$$
\ln (p / \mathrm{Pa})=(35.9005 \pm 0.9952)-(11239 \pm 317) /(T / K)
$$

sample II

$$
\ln (p / \mathrm{Pa})=(33.0469 \pm 0.6803)-(10513 \pm 218) /(T / K)
$$

which upon the appropriate differentiation yield enthalpies of sublimation of $\Delta_{\mathrm{cr}}^{\mathrm{g}} H_{\mathrm{m}}{ }^{\circ}(321.5 \mathrm{~K})=93.44 \pm 2.64 \mathrm{~kJ} \cdot \mathrm{mol}^{-1}$ and $\Delta_{\mathrm{cr}}^{g} H_{\mathrm{m}}{ }^{0}(319.2 \mathrm{~K})=87.41 \pm 1.81 \mathrm{~kJ} \cdot \mathrm{mol}^{-1}$ for samples $\mathrm{I}$ and
Table II. Vapor Pressure Data for 2,4,6-Trimethylbenzonitrile $N$-Oxide at Various Temperatures

\begin{tabular}{cccc}
\hline$T / \mathbf{K}$ & $p / \mathrm{Pa}$ & $10^{6} \mathrm{~m} / \mathrm{kg}$ & $t / \mathrm{s}$ \\
\hline \multicolumn{5}{c}{ Sample I: Original } & Sample as Received \\
300.33 & 0.0872 & 3.76 & 26688 \\
304.43 & 0.149 & 9.91 & 41454 \\
308.16 & 0.247 & 5.94 & 15054 \\
308.16 & 0.239 & 4.83 & 12654 \\
313.23 & 0.447 & 10.23 & 14454 \\
318.02 & 0.798 & 11.54 & 9204 \\
323.32 & 1.416 & 12.08 & 5476 \\
328.43 & 2.250 & 12.71 & 3654 \\
333.71 & 3.661 & 13.78 & 2454 \\
338.20 & 5.523 & 15.60 & 1854 \\
342.68 & 10.12 & 28.40 & 1854 \\
Sample II: & Purified by & & \\
299.95 & 0.153 & 5.87 & 23679 \\
304.33 & 0.216 & 6.33 & 18220 \\
308.16 & 0.364 & 6.72 & 11573 \\
312.40 & 0.542 & 8.19 & 9535 \\
316.78 & 0.845 & 9.98 & 7504 \\
321.25 & 1.355 & 16.26 & 7676 \\
325.11 & 2.084 & 12.14 & 3749 \\
329.32 & 3.093 & 11.72 & 2454 \\
333.46 & 4.507 & 22.57 & 3264 \\
338.38 & 7.250 & 20.43 & 1850
\end{tabular}

II. Sublimation enthalpies at the mean temperature can be corrected to $298.15 \mathrm{~K}$ assuming $\Delta_{\mathrm{cr}}^{g} C_{p}{ }^{\circ}=-R(20)$. For the purified sample, the computed sublimation enthalpy is $\Delta_{\mathrm{cr}}^{9} \mathrm{H}_{\mathrm{m}}{ }^{\circ}$ $=87.76 \pm 1.81 \mathrm{~kJ} \cdot \mathrm{mol}^{-1}$.

Not too much significance is placed on the first set of vapor pressure measurements because the sample became discolored at the elevated temperatures. To remove small impurities which may have resulted from prolonged shelf storage and/or $X$-ray inspections at airport security checkpoints, the original sample was purified by vacuum sublimation at $333 \mathrm{~K}$, as noted above. Vapor pressure measurements on sample II are believed to be reliable. The sublimed sample retained its white color over the entire temperature range examined. Combustion measurements were identical for both samples, at least within experimental uncertainty, and thus suggest that the degradation product was likely 2,4,6-trimethylbenzene isocyanate rather than a polymerization product. Our experimental enthalpy of sublimation is approximately $10 \mathrm{~kJ} \cdot \mathrm{mol}^{-1}$ more than the reported literature value of $\Delta_{\mathrm{cr}}^{\mathrm{g}} H_{\mathrm{m}}{ }^{\circ}=77.5 \pm 3.7 \mathrm{~kJ} \cdot \mathrm{mol}^{-1}(8)$ on the basis of vacuum sublimation drop microcalorimetry; however standard enthalpies of formation in the gaseous state are equivalent, $\Delta_{\mathrm{r}} H_{\mathrm{m}}{ }^{\circ}(\mathrm{g})=137.0 \pm 4.0 \mathrm{~kJ} \cdot \mathrm{mol}^{-1}$ versus $\Delta_{\mathrm{f}} H_{\mathrm{m}}{ }^{\circ}(\mathrm{g})$ $=133.4 \pm 4.0 \mathrm{~kJ} \cdot \mathrm{mol}^{-1}(8)$. Agreement between experimental values is considered excellent, particularly in light of the ther$\mathrm{mal} /$ photochemical instability of 2,4,6-trimethylbenzonitrile $\mathrm{N}$ oxide. Replicate, independent experimental determinations are a necessity whenever reactive compounds such as nitrile $N$ oxides are studied. Finally, our studies indicate that aromatic nitrile $N$-oxides do not necessarily exhibit the reported stability on shelf storage, and extreme care should be exercized in transporting/receiving samples for thermochemical measurements.

Reglstry No. 2,4,6-Trimethylbenzonitrile N-oxide, 2904-57-6. 


\section{Literature Clted}

(1) Hanhela, P. J.; Paul, D. B. Aust. J. Chem. 1989, 42, 287

(2) Hanhela, P. J.; Paul, D. B. Aust. J. Chem. 1989, 42, 1257.

(3) Grundmann, C.; Grunanger, P. The Nitrile Oxides; Springer-Verlag: Berlin, 1971.

(4) Torssell, K. B. G. Nitrile Oxides, Nitrones, and Nitronates in Organic Synthesis; VCH Publishers: New York, 1988; Chapter 2.

(5) Grundmann, C.; Dean, J. M. J. Org. Chem. 1965, 30, 2809.

(6) Christl, M.; Warren, J. P.; Hawkins, 8. L.; Roberts, J. D. J. Am. Chem. Soc. 1973, 95, 4392.

(7) Shiro, M.; Yamakawa, M.; Kobuta, T.; Koyama, H. J. Chem. Soc. Chem. Commun. 1968, 1409.

(8) Acree, W. E., Jr.; Tucker, S. A.; Zvaigzne, A. I.; Meng-Yan, Y.; PIlcher, G.; Ribeiro da Silva, M. D. M. C. J. Chem. Thermodyn. 1991, 23, 31.

(9) Beltrame, P.; Gelli, G.; Loi, A. Nouv. J. Chim. 1981, 5, 453.

(10) Simirsky, V. V.; Kabo, G. J.; Frenkel, M. L. J. Chem. Thermodyn. 1987, $19,1121$.
(11) Frenkel, M. L.; Kaler, G. V.; Simirskil, V. V.; Kabo, G. J. Russ. J. Phys. Chem. 1989, 63, 1253.

(12) Krasulin, A. P.; Kozyro, A. A.; Kabo, G. J. Zh. Prikl. Khim. 1987, 104

(13) Kozyro, A. A.; Frenkel, M. L.; Krasulln, A. P.; Simirskll, V. V.; Kabo, G. J. Russ. J. Phys. Chem. 1988, 62, 897

(14) Lavut, E. C.; Chelovskaya, N. V. Zh. Fiz. Khim. 1983, 57, 2108.

(15) Kornilov, A. N.; Volkova, I. M.; Skuratov, S. M. Zh. Fiz. Khim. 1964, $38,3035$.

(16) Bobransky, B. Qualitative Analysis of Organic Compounds; Nauka: Moscow, 1961.

(17) Alexsandrov, Y. I.; Mikina, V. D.; Novikov, G. A. Tr. Metrol. Inst SSSR Izd. Standartov, Moscow-Leningrad 1969, 171, 95.

(18) Rossini, F. D., Ed. Experimental Thermochemistry; Interscience Publishers: New York, 1956; Vol. 1.

(19) Alexsandrov, Y. I.; Oleynik, B. N.; Usvyatseva, T. R. Tr. Metrol. Inst SSSR Izd. Standartov, Moscow-Leningrad 1971, 189, 155.

(20) Sandman, D. J.; Epsteln, A. J.; Chickos, J. S.; Ketchum, J.; Fu, J. S.; Scheraga, H. A. J. Chem. Phys. 1979, 70, 305.

Received for review July 19, 1991. Accepted October 1, 1991.

\title{
Vapor Pressure, Density, Refractive Index, Excess Enthalpy, and Heat Capacity of 2-Chloro-2-methylpropane or Chlorobenzene + 2,2,4-Trimethylpentane
}

\author{
H. Kalall, F. Kohler, and P. Svejda* \\ Ruhr-Universität Bochum, Institut für Thermo- und Fluiddynamik, P.O. Box 102148, D-4630 Bochum 1, Germany
}

Excess volumes of the binary liquid mixtures of 2-chloro-2-methylpropane (tert-butyl chloride) or chlorobenzene $+2,2,4$-trimethylpentane (isooctane) have been determined at 293.15 and $313.15 \mathrm{~K}$ from density measurements at atmospherlc pressure. The excess enthalples, at $293.15 \mathrm{~K}$, and the excess isobaric heat capacties, at $293.15 \mathrm{~K}$ and $313.15 \mathrm{~K}$, have been measured using Plcker calorimeters at atmospheric pressure. Also the excess Gibbs energy of chlorobenzene + 2,2,4-trimethylpentane has been calculated from isothermal vapor-liquid equilibrium data at 303.15 and 323.15 K, taken in a dynamic Cottrell type apparatus, the equilibrium compositions being determined refractometrically.

\section{Introduction}

The measurements of the excess properties of 2-chloro-2methylpropane (tert-butyl chloride) or chlorobenzene $+2,2,4$ trimethylpentane (isooctane) presented here are part of a study on liquid mixtures of polar components (with different dipole moments) with a common nonpolar component, 2,2,4-trimethylpentane. References to other parts of this investigation have been summarized recently $(1,2)$.

2-Chloro-2-methylpropane and chlorobenzene are the least polar fluids in the series studied with dipole moments in the gas phase of 2.13 and $1.70 \mathrm{D}$, leading to reduced dipole moments of 0.695 and $0.494(2)$, respectively.

\section{Experimental Section}

Materlals. 2-Chloro-2-methylpropane (EGA-Chemie, p. a.) with a purity of $98.80 \mathrm{~mol} \%$ and chlorobenzene (Fluka, puriss.) with a purity of $99.98 \mathrm{~mol} \%$, both checked by GLC, were dried by storage over calcium chloride and over potassium carbonate, respectively (J. T. Baker Chemicals, Analytical Reagent).
2,2,4-Trimethylpentane (Fluka, puriss., or Phillips, Research Grade) with GLC purities of 99.97 or $99.98 \mathrm{~mol} \%$, respectively, was stored over $\mathrm{Na}-\mathrm{Pb}$ (Merck, p. a.). The substances were used without further purification. They were carefully degassed by several pump/thaw cycles prior to each measurement. The molar quantities were calculated using the relative atomic masses according to IUPAC (1987) (3).

In Table I, the experimental densities $\rho$, the molar isobaric heat capacities $C_{p}$ and, if measured, the vapor pressures $p$ and the refractive indices $n_{D}$ of the pure liquids are compared with literature data.

Apparatus and Procedure. Molar excess volumes $V^{E}$ were calculated from precise density measurements of the liquids measured by means of a vibrating glass tube densimeter (Model DMA 02 D, Paar) at 293.15 and $313.15 \mathrm{~K}$ and atmospheric pressure. The precision in the densities is estimated as $3 \times$ $10^{-6} \mathrm{~g} \mathrm{~cm}^{-3}$, the accuracies are $2 \times 10^{-5} \mathrm{~g} \mathrm{~cm}^{-3}$, at 293.15 $\mathrm{K}$, and $1 \times 10^{-4} \mathrm{~g} \mathrm{~cm}^{-3}$, at $313.15 \mathrm{~K}$. $V^{\mathrm{E}}$ is accurate to 0.003 $\mathrm{cm}^{3} \mathrm{~mol}^{-1}$ at about equimolar composition. Mixtures were prepared from the degassed samples under vacuum by weighing them into airtight screw-capped tubes; the masses were corrected for the gas phase. This led to accuracies in the mole fraction $x_{i}$ of $3 \times 10^{-5}$. Details are given in ref 4 .

The molar excess enthalpies $H^{E}$ (at $293.15 \mathrm{~K}$ ) and the molar excess isobaric heat capacities $C_{p}{ }^{\mathrm{E}}$ (at 293.15 and $313.15 \mathrm{~K}$ ) at atmospheric pressure were measured with a dynamic flow and a differential flow microcalorimeter, respectively, of Picker type (Setaram). At equimolar composition, $H^{E}$ could be measured with a relative error of less than $2.5 \%$. The absolute molar $C_{p}$ values of 2,2,4-trimethylpentane were estimated to be accurate to $1.2 \mathrm{~J} \mathrm{~mol}^{-1} \mathrm{~K}^{-1}$; the errors for $C_{p}$ of 2-chloro-2methylpropane and chlorobenzene were estimated to be $1 \%$. In a stepwise procedure, only small differences of the volumetric heat capacities were measured from one concentration to another one over the entire range, for which uncertaintles in the heat capacity of the pure compounds did not matter; from this $C_{p}{ }^{E}$ could be determined with an accuracy of $0.02 \mathrm{~J} \mathrm{~mol}^{-1}$ 